\title{
Comparison and exploration of the prognostic value of the advanced lung cancer inflammation index, prognostic nutritional index, and systemic immune-inflammation index in newly diagnosed diffuse large B-cell lymphoma
}

\author{
Tingting Liu, Fan Ye, Yuanyuan Li, Aining Liu \\ Hematology Department, Affiliated Hospital of Jiangnan University, Wuxi, China \\ Contributions: (I) Conception and design: T Liu, A Liu; (II) Administrative support: T Liu, A Liu; (III) Provision of study materials or patients: T Liu, \\ F Ye, Y Li; (IV) Collection and assembly of data: T Liu, F Ye, Y Li; (V) Data analysis and interpretation: T Liu, A Liu; (VI) Manuscript writing: All \\ authors; (VII) Final approval of manuscript: All authors. \\ Correspondence to: Aining Liu. Hematology Department, Affiliated Hospital of Jiangnan University, 1000 Hefeng Road, Binhu District, Wuxi 214026, \\ China. Email: 13961629559@163.com.
}

\begin{abstract}
Background: The international prognostic index (IPI) is widely used as an indicator for evaluating the clinical prognosis of diffuse large B-cell lymphoma (DLBCL). However, more precise prognostic indicators are needed. This study aimed to evaluate the prognostic significance of the advanced lung cancer inflammation index (ALI), prognostic nutritional index (PNI), and systemic immune-inflammation index (SII) in DLBCL.
\end{abstract}

Methods: A total of 117 patients with newly diagnosed DLBCL were included in this study. Receiver operating characteristic (ROC) curve analysis was used to determine the optimal cut-off values for the ALI, PNI, and SII to predict survival, and patients were stratified into high and low groups. Cox regression analysis and the Kaplan-Meier method were used to assess the prognostic ability of these indexes.

Results: The optimal cut-offs for the ALI, PNI, and SII were 31.26, 36.48, and 486.76, respectively. The ALI had the highest area under the curve (AUC). The high ALI or PNI group had better 5-year overall survival (OS) than the low ALI (73\% vs. $53 \%, \mathrm{P}<0.001)$ or $\mathrm{PNI}(60 \%$ vs. $45 \%, \mathrm{P}<0.001)$ group, and the low SII group had better 5 -year OS than the high SII group $(67 \%$ vs. 62\%, P=0.034). Although all 3 parameters were associated with OS in univariate analyses, only the ALI and PNI were independent factors for OS in multivariate analyses. We found that when DLBCL patients were classified according to IPI combined with ALI, PNI, or SII, respectively, there were more obvious differences in OS among different types.

Conclusions: The ALI and PNI may be easily available markers to predict clinical outcomes in DLBCL patients. SII predicted OS only in univariate analysis.

Keywords: Advanced lung cancer inflammation index (ALI); systemic immune-inflammation index (SII); prognostic nutritional index (PNI); newly diagnosed diffuse large B-cell lymphoma (NDDLBCL)

Submitted Jul 02, 2021. Accepted for publication Sep 09, 2021.

doi: 10.21037/apm-21-2067

View this article at: https://dx.doi.org/10.21037/apm-21-2067

\section{Introduction}

Diffused large B-cell lymphoma (DLBCL) accounts for $25-35 \%$ of non-Hodgkin's lymphoma and is the most common invasive lymphoma in clinical practice
$(1,2)$. As a first-line chemotherapy regimen, $\mathrm{R}-\mathrm{CHOP}$ (rituximab, cyclophosphamide, doxorubicin, vincristine, and prednisone) has significantly improved patient outcomes. However, approximately $20 \%$ of all patients have primary refractory disease, and approximately $30 \%$ of all patients 
relapse $(3,4)$. Therefore, at the time of initial diagnosis, it is very important to stratify the prognosis of DLBCL patients and individualize treatment according to their stratification. Patients with poor prognosis may require more intense or longer chemotherapy. The International Prognostic Index (IPI) is the most widely used indicator for evaluating the clinical prognosis of DLBCL $(5,6)$; however, some patients with a good IPI still fail treatment and some with a poor IPI have good response. The prognostic value of the IPI scoring system is based on the era of traditional chemotherapy drugs. However, in the new era of rituximab and other targeted drugs as first-line therapy, and the application of cell origin or genomics analysis technology in clinical practice, the prognostic value of IPI is gradually decreasing. Hence, more precise prognostic indicators are needed.

Previous studies have reported that blood cell counts such as lymphocyte/monocyte ratio (7) and systemic immuneinflammation index (SII) (8) can provide guiding value for the prognosis of DLBCL patients. Recently, it has been reported that low prognostic nutritional index (PNI) is an indicator of poor prognosis in DLBCL patients (9). Jafri et al. (10) developed a new prognostic index called the advanced lung cancer inflammation index (ALI). Park et al. showed that a low pre-treatment ALI $(<15.5)$ indicated poor response to chemotherapy in DLBCL patients treated with R-CHOP, and poor prognosis (11). However, which indicator is the most reliable prognostic factor for DLBCL remains to be clarified. Therefore, the purpose of this study was to validate and compare the prognostic value of the ALI, PNI, and SII for newly diagnosed DLBCL. We present the following article in accordance with the STARD reporting checklist (available at https://dx.doi.org/10.21037/apm-21-2067).

\section{Methods}

\section{Patients}

Our study collected the data of 128 DLBCL patients who were initially diagnosed at the Affiliated Hospital of Jiangnan University in China between March 2011 and August 2019. After reconfirming the immunohistochemistry (IHC), 5 patients were excluded due to unclear IHC. Data were missing for 6 patients, and 117 were eventually analyzed.

The study was conducted in accordance with the Declaration of Helsinki (as revised in 2013). The study was approved by the Medical Science Research Ethics Committee of the Affiliated Hospital of Jiangnan University and individual consent for this retrospective analysis was waived (No. LS 2020065).

Demographic and disease findings at the first presentation and before treatment, including age, gender, Eastern Cooperative Oncology Group (ECOG) performance status, histologic subtype, presence of B symptoms, height, weight, and laboratory test data, including blood routine analysis, lactate dehydrogenase (LDH), albumin, $\beta 2$ microglobulin (B2M) levels, C-reactive protein (CRP), D-dimer, and total cholesterol (TC), were obtained from hospital records. The IPI scoring was conducted according to patients' age, ECOG performance status (PS), extranodal involvement, serum LDH, and Ann Arbor (AA) system disease stage.

The following calculations were used to arrive sat the relative values:

* NLR: peripheral blood levels of absolute neutrophil count/absolute lymphocyte count.

- ALI: body mass index $(\mathrm{BMI}) \times$ blood albumin level $(\mathrm{g} / \mathrm{dL}) / \mathrm{NLR}$.

* PNI: $10 \times$ serum albumin $(\mathrm{g} / \mathrm{dL})+0.005 \times$ total lymphocyte count $/ \mathrm{mm}^{3}$.

* SII: platelet count $\times$ NLR.

ALI, PNI, SII area under the curve (AUC), sensitivity, specificity and Yoden index (sensitivity + specificity -1 ) were derived from the ROC curve. The maximum value of the Youden index was considered as the optimal cut-off value.

All participants were followed from the date of diagnosis until death or cutoff time, and overall survival (OS) was calculated.

\section{Statistical analysis}

According to the cut-off value, the population was divided into high/low ALI, high/low PNI, or high/low SII groups. Chi-square $\left(\chi^{2}\right)$ test and Fisher's exact test were used to analyze the differences of clinicopathological variables between groups with high/low ALI, high/low PNI, and high/ low SII, respectively. Univariate and multivariate analyses were performed using the Cox proportional risk regression model to evaluate prognostic factors and their impact on OS. Survival curves were drawn using Kaplan Meier curve analysis, and we used the log-rank test for comparison. A P value $<0.05$ was considered statistically significant.

\section{Results}

\section{Participants}

The study flow chart is shown in Figure 1. A total of 


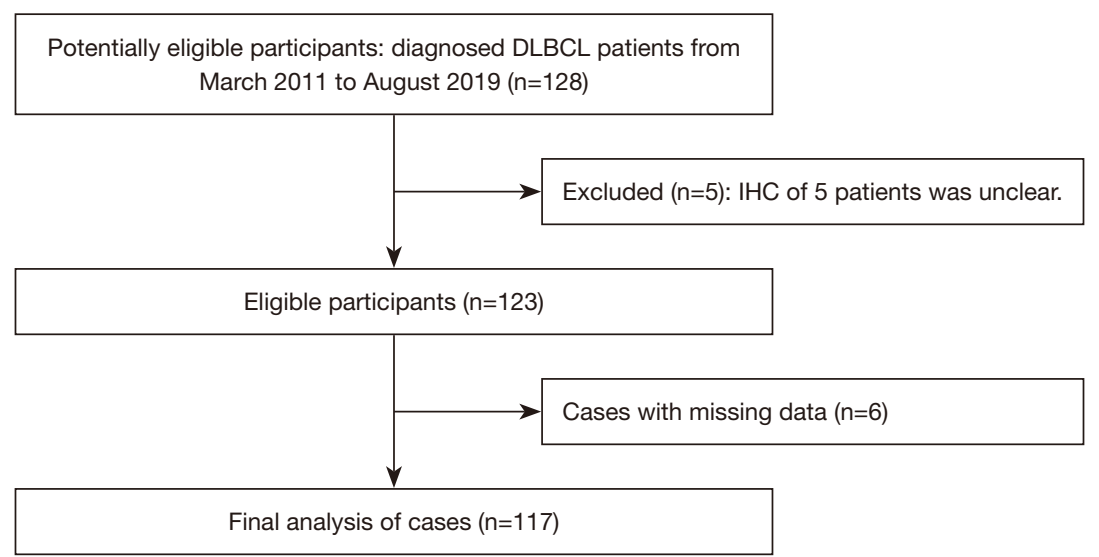

Figure 1 Flow chart of the study. DLBCL, diffuse large B-cell lymphoma; IHC, immunohistochemistry.

128 participants were included in this retrospective cohort study. After reconfirming the IHC, 5 patients were excluded from the group on account of unclear IHC. Data were missing for 6 patients, and 117 finally included in the analysis.

The characteristics of 117 participants are shown in Table 1. A total of 55 patients were female, and the median age was 63 years (20-83 years). A total of 64 (54.7\%) participants had advanced disease (AA stage III-IV). The IPI score of 38 participants $(32.47 \%)$ was greater than or equal to 3 points. A total of $61(52.14 \%)$ participants had a histologically confirmed germinal center B-cell (GCB) subtype and $56(47.86 \%)$ had a histologically confirmed non-GCB subtype. All patients received CHOP/R-CHOP/ R-CHOPE chemotherapy for at least 3 cycles. The median follow-up time was 39 [15-178] months, and the 3-year and 5 -year OS rates were $69 \%$ and $64 \%$, respectively.

\section{ROC curve analysis}

The most sensitive and specific cut-off values for the ALI, PNI, and SII were $31.26,36.48$, and 486.76, respectively (Figure 2). The ALI had the highest area under the curve (AUC, 0.675; sensitivity, 65.1\%; specificity, 67.6\%).

\section{Correlation of the ALI, PNI, and SII with clinical characteristics}

Based on the cutoff of ALI, PNI, and SII, all participants were divided into high or low ALI, PNI, and SII groups. Participants with a low ALI or PNI had a higher incidence of anemia and lower cholesterol than those with a high ALI or PNI. Participants with a low ALI or PNI or high SII had significantly higher mortality, CRP, LDH, D-dimer, and WBC than those in the high ALI or PNI or low SII group (Table 1). Participants with a low ALI or high SII had B symptoms more frequently than those with the high ALI or a low SII (Table 1).

Univariate analysis showed that, among the clinical indicators, stage, age, IPI score, ALI, PNI, and SII were predictive of OS. However, in multivariate analysis, only the IPI score, ALI, and PNI were risk factors for OS (Table 2).

\section{Survival effects of the ALI, PNI, and SII}

Log-rank tests showed that the 5-year OS in the high ALI group, high PNI group, or low SII group were higher than that in the low ALI group (73\% vs. $53 \%, \chi^{2}=11.134$, $\mathrm{P}=0.001)$, the low PNI group $\left(60 \%\right.$ vs. $45 \%, \chi^{2}=17.932$, $\mathrm{P}<0.001)$, or the high SII group (67\% vs. $62 \%, \chi^{2}=4.514$, $\mathrm{P}=0.034)$, respectively (Figure 3).

An IPI score of 0-2 was classified as the low IPI group, and an IPI score of 3 or more was classified as the high IPI group. The 5 -year OS of the high IPI group was $44 \%$, and that of the low IPI group was $73 \%\left(\chi^{2}=18.176, \mathrm{P}<0.001\right)$. Combined with ALI and IPI indexes, they were divided into 3 groups: low ALI group + high IPI; high ALI + high IPI or low ALI + low IPI; and high ALI + low IPI group. The 5 -year OS of the 3 groups were $37 \%, 62 \%$, and $78 \%$ $\left(\chi^{2}=24.232, \mathrm{P}<0.001\right)$, respectively. The PNI combined with IPI were divided into low PNI + high IPI group; High PNI + high IPI or low PNII + low IPI; and high PNI + low IPI group. The respective 5 -year OS rates were $19 \%, 60 \%$, and $75 \%\left(\chi^{2}=41.922, \mathrm{P}<0.001\right)$. According to SII combined with 
Table 1 Baseline participant, characteristics of ALI, PNI, and SII

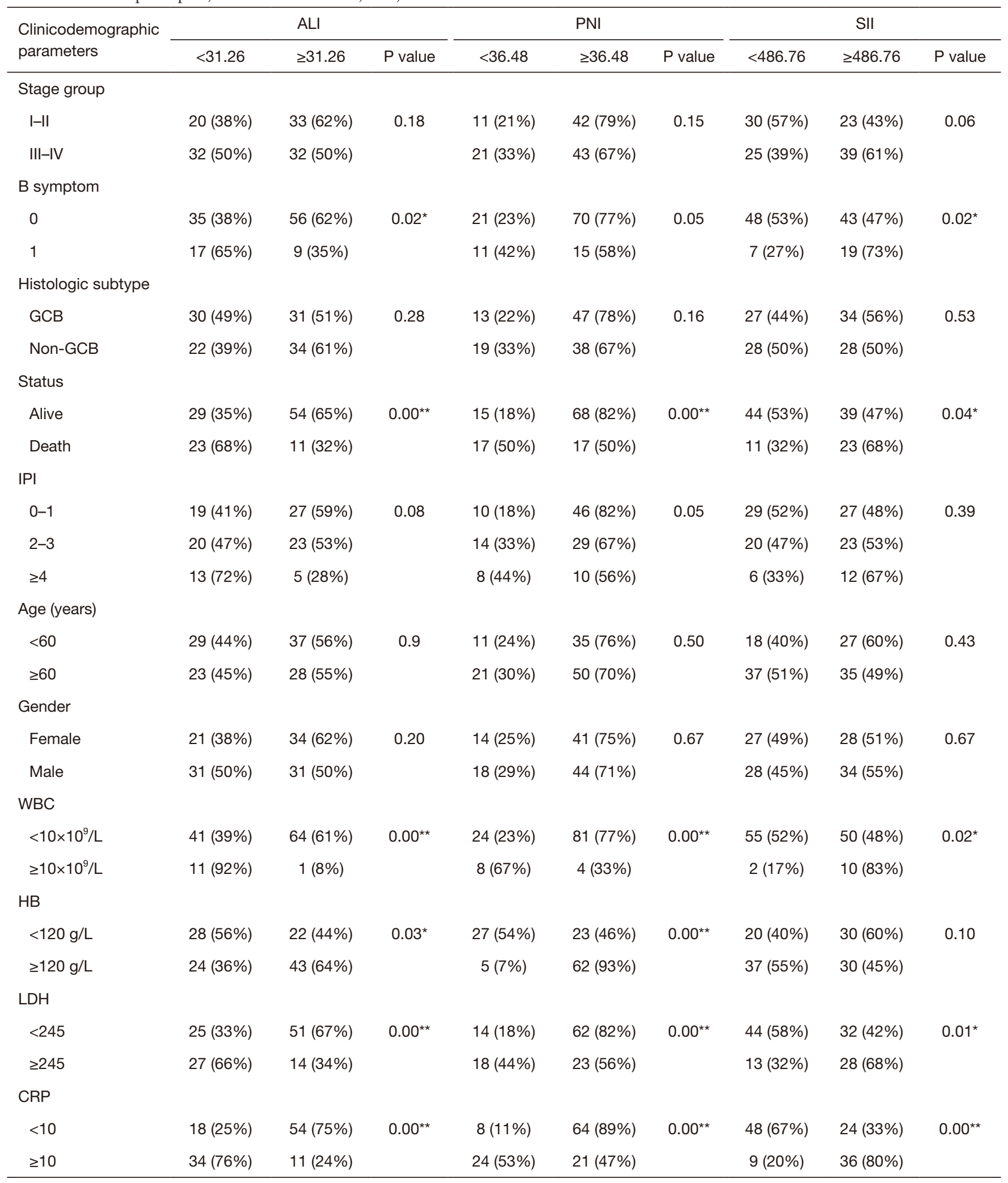

Table 1 (continued) 
Table 1 (continued)

\begin{tabular}{|c|c|c|c|c|c|c|c|c|c|}
\hline $\begin{array}{l}\text { Clinicodemographic } \\
\text { parameters }\end{array}$ & \multicolumn{3}{|c|}{ ALI } & \multicolumn{3}{|c|}{ PNI } & \multicolumn{3}{|c|}{ SII } \\
\hline \multicolumn{10}{|l|}{ Albumin } \\
\hline$<40$ & 52 (85\%) & 9 (15\%) & $0.00^{\star \star}$ & 30 (51\%) & $29(49 \%)$ & $0.00^{\star *}$ & 24 (39\%) & 37 (61\%) & $0.03^{*}$ \\
\hline$\geq 40$ & $0(0 \%)$ & 56 (100\%) & & $2(3 \%)$ & 56 (97\%) & & 33 (59\%) & $23(41 \%)$ & \\
\hline$<3$ & 41 (39\%) & $63(61 \%)$ & $0.00^{\star \star}$ & $24(23 \%)$ & $80(77 \%)$ & $0.01^{\star *}$ & 52 (49\%) & 55 (51\%) & 0.93 \\
\hline$\geq 3$ & $11(85 \%)$ & 2 (15\%) & & $8(62 \%)$ & 5 (38\%) & & 5 (50\%) & 5 (50\%) & \\
\hline \multicolumn{10}{|l|}{ D-dimer } \\
\hline$<0.5$ & $11(24 \%)$ & 35 (76\%) & $0.00^{\star \star}$ & $4(9 \%)$ & 42 (91\%) & $0.00^{\star \star}$ & 29 (63\%) & 17 (37\%) & $0.01^{\star *}$ \\
\hline$<3.5$ & 24 (62\%) & 15 (38\%) & $0.01^{\star *}$ & 15 (39\%) & $23(61 \%)$ & $0.04^{\star}$ & $16(41 \%)$ & 23 (59\%) & 0.24 \\
\hline$\geq 3.5$ & $28(36 \%)$ & 50 (64\%) & & 17 (22\%) & 62 (78\%) & & $41(53 \%)$ & $37(47 \%)$ & \\
\hline
\end{tabular}

*, $\mathrm{P}<0.05 ;{ }^{* \star}, \mathrm{P} \leq 0.01$. GCB, germinal center B-cell; TC, total cholesterol; LDH, lactate dehydrogenase; WBC, white blood cell; ALI, advanced lung cancer inflammation index; PNI, prognostic nutritional index; SII, systemic immune-inflammation index; B2M, $\beta 2$ microglobulin; $\mathrm{Hb}$, hemoglobin; CRP, C-reactive protein.

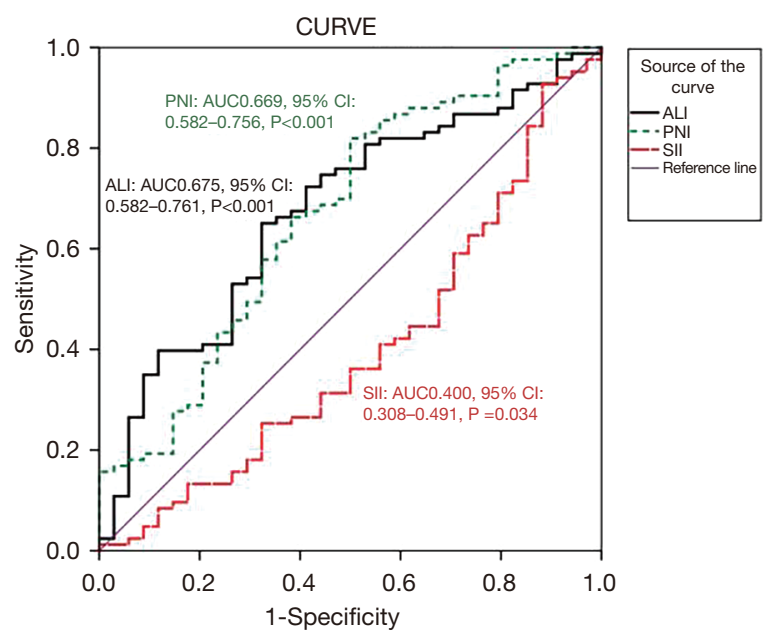

Figure 2 ROC analysis and AUC for sensitivity and specificity of parameters. ROC, receiver operating characteristic; AUC, area under the curve; ALI, advanced lung cancer inflammation index; PNI, prognostic nutritional index; SII, systemic immuneinflammation index.

IPI, the patients were divided into high SII + high IPI, low SII + high IPI or high SII + low IPI, and low SII + low IPI, and their 5 -year OS were $45 \%, 64 \%$, and $75 \%\left(\chi^{2}=17.254\right.$,
$\mathrm{P}<0.001)$ (Figure 4).

\section{Discussion}

In our study, we analyzed the prognostic values of 3 different indicators, ALI, PNI, and SII, in newly diagnosed DLBCL. Although all 3 parameters were successful in stratifying patients into 2 prognostic groups, only the ALI and PNI were found to be independent risk factors for OS. Among the 3, the ALI had the highest AUC. We also found that patients grouped by IPI combined with ALI, PNI, or SII had more significant differences in OS between the groups.

Inflammation and nutrition play significant roles in tumor progression (12). Park et al. (13) reported that malnutrition is an adverse prognostic factor in DLBCL patients. Inflammation plays an important role in the pathogenesis and progression of several types of lymphoma, including DLBCL, and there are varying levels of inflammatory infiltrates, including macrophages, and effector and regulatory $\mathrm{T}$ cells (14). Due of this association, multiple systemic nutritional and inflammatory biomarkers have been studied in DLBCL patients to predict prognosis. These biomarkers include the PNI (9), GNRI (15), NLR (16), 
Table 2 Univariate and multivariate analysis of IPI system for OS

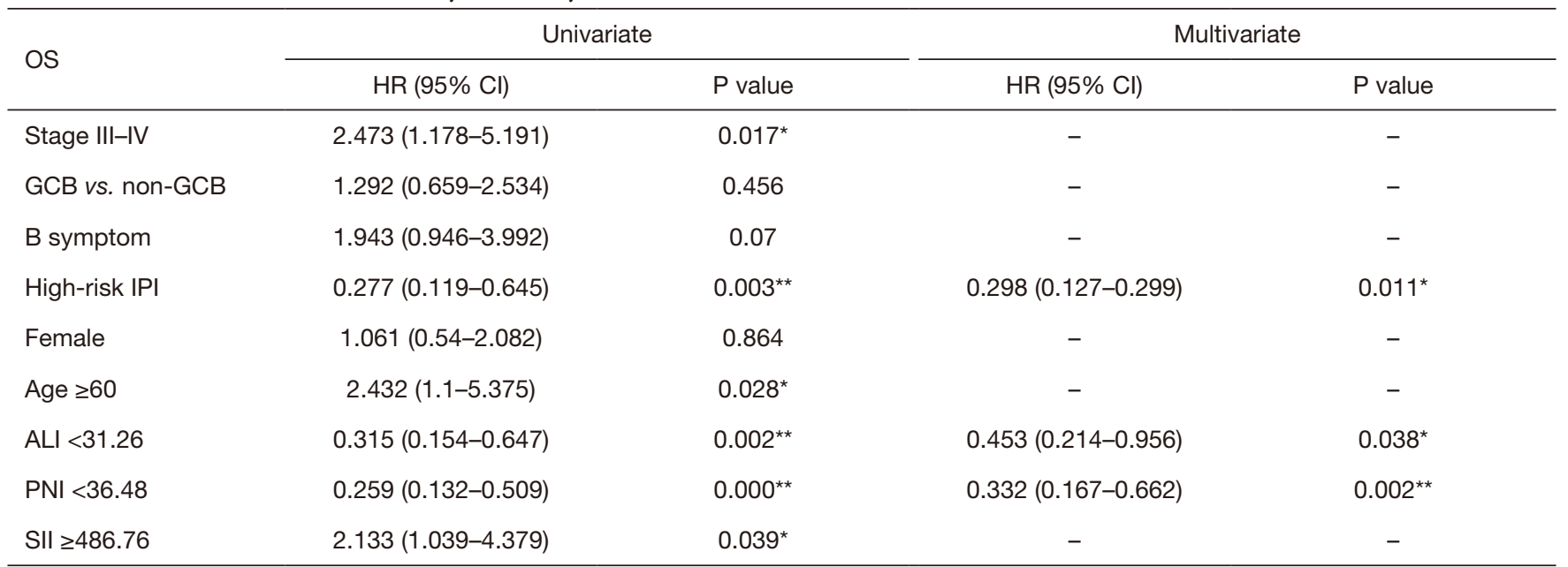

*, $\mathrm{P}<0.05 ;{ }^{*}, \mathrm{P} \leq 0.01$. IPI, international prognostic index; OS, overall survival; GCB, germinal center B-cell; ALI, advanced lung cancer inflammation index; PNI, prognostic nutritional index; SII, systemic immune-inflammation index; HR, hazard ratio; $\mathrm{Cl}$, confidence interval.

A

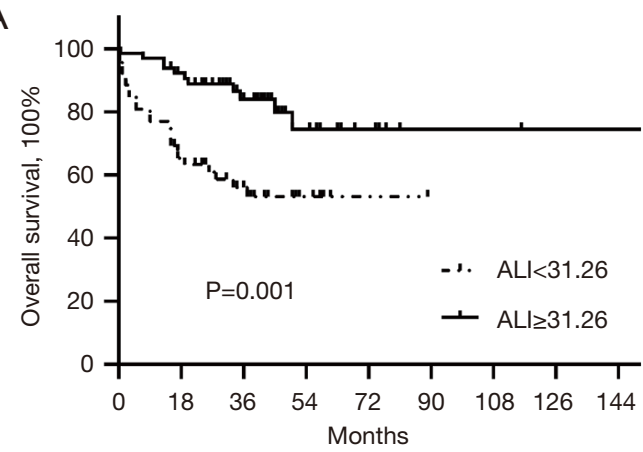

C

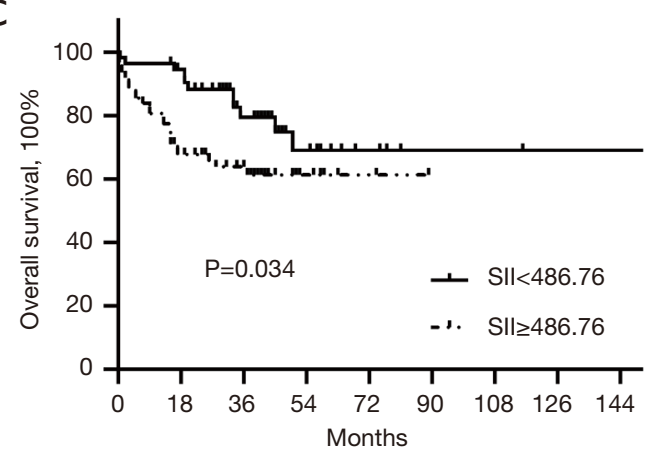

B

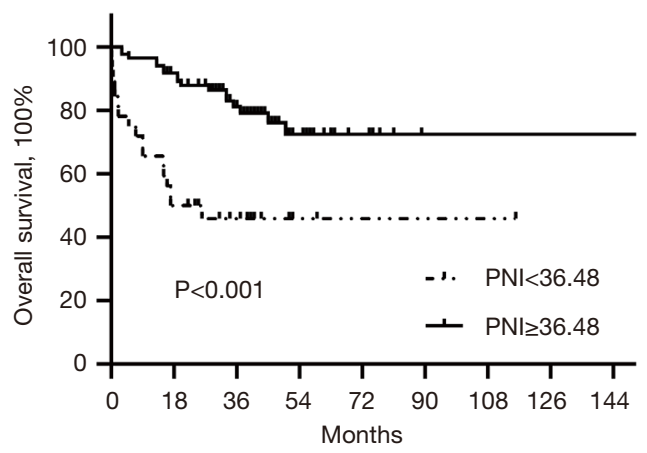

Figure 3 OS according to ALI (A), PNI (B), and SII (C). OS, overall survival; ALI, advanced lung cancer inflammation index; PNI, prognostic nutritional index; SII, systemic immune-inflammation index.

GPS (17), and peripheral blood absolute lymphocyte and monocyte counts (18). Based on the numerous clinical composite indicators reported in the literature, we examined the prognostic value of the ALI, PNI, and SII.
The ALI is considered a better indicator of systemic inflammation than current biomarkers. A low ALI at diagnosis is associated with poor prognosis in esophageal cancer and lung cancer patients $(10,19,20)$. Park et al. 

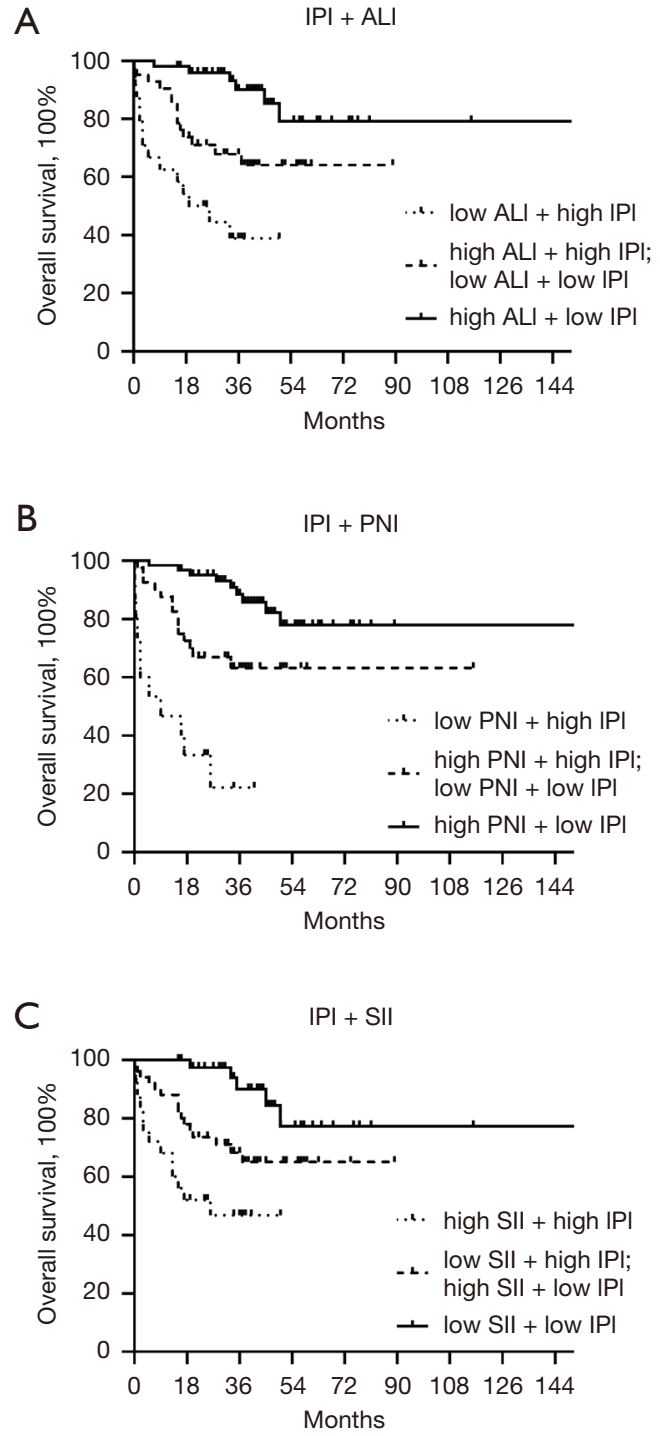

Figure 4 OS according to IPI + ALI (A), IPI + PNI (B), IPI + SII (C). OS, overall survival; IPI, international prognostic index; ALI, advanced lung cancer inflammation index; PNI, prognostic nutritional index; SII, systemic immune-inflammation index.

reported that in DLBCL, a low ALI was associated with lower rates of complete remission and poorer 5-year OS (11). In this study, the incidence of B symptoms, inflammatory indicators such as WBC count, or indicators reflecting tumor burden such as $\mathrm{LDH}$ were higher in the low ALI group. The levels of HB in the low ALI group were also lower than those in the other group. However, in 2 groups of patients, common clinical prognostic indicators such as installment, germinal center origin, and IPI score had no significant differences. This result differed from that reported by Park et al. (11), who indicated that age, stage, and IPI score were different between high and low ALI patients, whereas LDH, B2M, CRP, and other indicators were not significantly different. We found that the OS in the low ALI group was lower, which is similar to previously reported results (11).

Initially, PNI used to evaluate the nutritional and surgical risks of patients undergoing gastrointestinal surgery $(21,22)$. Later, it was found to be a simple and effective predictor of the prognosis of a variety of diseases, including solid tumors and hematological diseases. Previous studies have revealed that PNI can effectively predict the prognosis of DLBCL $(23,24)$. This study showed that the OS in the low PNI group was lower, similar to what was observed for the ALI. The inflammatory indexes and tumor load indexes were higher in the low PNI group, which was consistent with the results of Periša et al. (25) and Go et al. (23). However, there were no differences between the 2 groups in clinical indicators such as age, B symptoms, stage, and IPI score.

The SII, a relatively new inflammatory index, combines the neutrophil, lymphocyte, and platelet counts. The SII reflects the inflammatory status and correlates with circulating tumor cells. Platelets have important properties not only in angiogenesis but also in tumor cell immune evasion and extravasation to other organs (26). High SII in patients with hepatocellular carcinoma, renal cell carcinoma, gastrointestinal tract cancers, and small cell lung cancer have been found to be associated with worse OS (27-30). Although there is growing evidence of the importance of SII in solid tumors, there are limited data on SII in hematopoietic tumors. In hematologic tumors, the SII has been examined only in Hodgkin lymphoma (HL) (31) and testicular DLBCL (8). In this study, we analyzed the role of the SII in newly diagnosed DLBCL without distinguishing specific site. In univariate analysis, the SII was significantly associated with the risk of disease progression $(\mathrm{P}=0.005)$, but it was not significant in multivariate analysis, which is consistent with the findings of Yang et al. (8). However, the AUC for the SII in our study was not high. Therefore, a larger sample may be needed to validate the clinical significance of SII for non-specific site of DLBCL.

This study did not identify differences between the low and high ALI, PNI, and SII groups in staging, IPI score, or germinal center origin. This may be related to the limited number of participants. It is also possible that, in the current era of targeted therapies such as rituximab, new drugs may be able to overcome the differences in the origin of tumors, and more attention should be paid to genetic and molecular 
abnormalities at the time of initial diagnosis.

The D-dimer was different in the high and low ALI, PNI, and SII groups. It has been reported that plasma D-dimer plays an important role in predicting disease progression and prognosis in malignant tumor patients $(32,33)$; however, there is little analysis of $\mathrm{D}$-dimer in lymphoma. In a cohort study, elevated D-dimer levels were associated with poorer OS in natural killer/T-cell lymphoma patients, and higher D-dimer levels were an independent predictor of poor OS (34). In this study, we found that D-dimer also differed between each group.

Population-based studies have reported that high circulating TC concentration is associated with lower cancer incidence and mortality (35). Here, we found that TC was lower in the high ALI, high PNI, and low SII groups than in the low ALI, low PNI, and high SII groups, respectively, indicating that TC can reflect nutritional status. It can be inferred that the nutritional status of patients with high TC should be better than that of those with low TC. However, it has been reported that low TC level is an adverse prognostic factor in the rituximab era (36); therefore, our results require further confirmation by a larger sample.

Of the ALI, PNI, and SII, the ALI had the highest AUC. The ALI is calculated using the BMI, albumin levels, and NLR. Some studies have shown that malnutrition can be determined by non-voluntary weight loss and low BMI in $94.1 \%$ of patients (37). Serum albumin, as an important nutritional indicator, is produced by the liver and plays an important role in improving the body's immunity, inflammatory state, and anti-tumor activity. More aggressive tumor cells can catabolize albumin more rapidly. Various factors secreted by the tumor microenvironment, such as interleukin-6, interferon $\gamma$, and CXCL-10, can promote cell proliferation, migration, and invasion. These cytokines are capable of inhibiting lymphocyte production and stimulating neutrophil production $(38,39)$. Hence, the NLR reflects the inflammatory condition and is also a powerful indicator of systemic inflammation in cancer (16). Therefore, the ALI is a more comprehensive indicator than the PNI and SII and has a better predictive effect on the prognosis of DLBCL. However, this conclusion may need to be examined in a larger sample study.

The IPI is currently widely used in clinical practice. We, therefore, aimed to combine ALI, PNI, and SII with IPI, and found that the combined indicators could more comprehensively classify patients, and their prognoses were also significantly stratified. This indicates that in future clinical work, we can better predict the prognosis of
DLBCL patients based on IPI combined with ALI, PNI, and SII indicators.

This study had several limitations. First, the study involved a relatively small sample size, which may have led to bias in the research results. Second, we only assessed the status of ALI, PNI, and SII at baseline, and there was a lack of longitudinal monitoring and evaluation of indicators. Third, molecular tests such as fluorescence in situ hybridization were not completed in many participants; therefore, further stratification of prognostic risk was not possible. Fourth, we only evaluated OS and did not examine PFS.

In the new era of targeted drugs and genome sequencing, simple, easy to obtain, stable and comprehensive biological markers will be necessary tools to evaluate the prognosis of DLBCL and guide the choice of treatment plan for patients. Our study supports that indicators such as ALI, PNI and SII, can comprehensively evaluate all aspects of the patient's situation, suggesting their use as promising markers for future prognoses.

In conclusion, this study shows that ALI and PNI can effectively predict the prognosis of patients with DLBCL, and it is convenient and simple. The SII, although not clearly shown to be an independent risk factor, was reported for the first time in non-specific site DLBCL patients. Moreover, combined ALI, PNI, and SII on the basis of IPI score can play a more effective role in predicting the prognosis. However, these conclusions need to be confirmed through larger sample studies.

\section{Acknowledgments}

Funding: None.

\section{Footnote}

Reporting Checklist: The authors have completed the STARD reporting checklist. Available at https://dx.doi. org/10.21037/apm-21-2067

Data Sharing Statement: Available at https://dx.doi. org/10.21037/apm-21-2067

Conflicts of Interest: All authors have completed the ICMJE uniform disclosure form (available at https://dx.doi. org/10.21037/apm-21-2067). The authors have no conflicts of interest to declare.

Ethical Statement: The authors are accountable for all 
aspects of the work in ensuring that questions related to the accuracy or integrity of any part of the work are appropriately investigated and resolved. The study was conducted in accordance with the Declaration of Helsinki (as revised in 2013). The study was approved by the Medical Science Research Ethics Committee of Affiliated Hospital of Jiangnan University and individual consent for this retrospective analysis was waived (NO.LS 2020065).

Open Access Statement: This is an Open Access article distributed in accordance with the Creative Commons Attribution-NonCommercial-NoDerivs 4.0 International License (CC BY-NC-ND 4.0), which permits the noncommercial replication and distribution of the article with the strict proviso that no changes or edits are made and the original work is properly cited (including links to both the formal publication through the relevant DOI and the license). See: https://creativecommons.org/licenses/by-nc-nd/4.0/.

\section{References}

1. Sabattini E, Bacci F, Sagramoso C, et al. WHO classification of tumours of haematopoietic and lymphoid tissues in 2008: an overview. Pathologica 2010;102:83-7.

2. Morton LM, Wang SS, Devesa SS, et al. Lymphoma incidence patterns by WHO subtype in the United States, 1992-2001. Blood 2006;107:265-76.

3. Coiffier B, Lepage E, Briere J, et al. CHOP chemotherapy plus rituximab compared with CHOP alone in elderly patients with diffuse large-B-cell lymphoma. $\mathrm{N}$ Engl J Med 2002;346:235-42.

4. Pfreundschuh M, Trümper L, Osterborg A, et al. CHOPlike chemotherapy plus rituximab versus CHOPlike chemotherapy alone in young patients with goodprognosis diffuse large-B-cell lymphoma: a randomised controlled trial by the MabThera International Trial (MInT) Group. Lancet Oncol 2006;7:379-91.

5. Sehn LH, Berry B, Chhanabhai M, et al. The revised International Prognostic Index (R-IPI) is a better predictor of outcome than the standard IPI for patients with diffuse large B-cell lymphoma treated with R-CHOP. Blood 2007;109:1857-61.

6. Zhou Z, Sehn LH, Rademaker AW, et al. An enhanced International Prognostic Index (NCCN-IPI) for patients with diffuse large B-cell lymphoma treated in the rituximab era. Blood 2014;123:837-42.

7. Bento L, Díaz-López A, Barranco G, et al. New prognosis score including absolute lymphocyte/monocyte ratio, red blood cell distribution width and beta-2 microglobulin in patients with diffuse large B-cell lymphoma treated with R-CHOP: Spanish Lymphoma Group Experience (GELTAMO). Br J Haematol 2020;188:888-97.

8. Yang J, Guo X, Hao J, et al. The Prognostic Value of Blood-Based Biomarkers in Patients With Testicular Diffuse Large B-Cell Lymphoma. Front Oncol 2019;9:1392.

9. Luan C, Wang F, Wei N, et al. Prognostic nutritional index and the prognosis of diffuse large b-cell lymphoma: a meta-analysis. Cancer Cell Int 2020;20:455.

10. Jafri SH, Shi R, Mills G. Advance lung cancer inflammation index (ALI) at diagnosis is a prognostic marker in patients with metastatic non-small cell lung cancer (NSCLC): a retrospective review. BMC Cancer 2013;13:158.

11. Park YH, Yi HG, Lee MH, et al. Prognostic Value of the Pretreatment Advanced Lung Cancer Inflammation Index (ALI) in Diffuse Large B Cell Lymphoma Patients Treated with R-CHOP Chemotherapy. Acta Haematol 2017;137:76-85.

12. Zitvogel L, Pietrocola F, Kroemer G. Nutrition, inflammation and cancer. Nat Immunol 2017;18:843-50.

13. Park S, Han B, Cho JW, et al. Effect of nutritional status on survival outcome of diffuse large B-cell lymphoma patients treated with rituximab-CHOP. Nutr Cancer 2014;66:225-33.

14. Carbone A, Tripodo C, Carlo-Stella C, et al. The role of inflammation in lymphoma. Adv Exp Med Biol 2014;816:315-33.

15. Lee S, Fujita K, Morishita T, et al. Prognostic utility of a geriatric nutritional risk index in combination with a comorbidity index in elderly patients with diffuse large $\mathrm{B}$ cell lymphoma. Br J Haematol 2021;192:100-9.

16. Keam B, Ha H, Kim TM, et al. Neutrophil to lymphocyte ratio improves prognostic prediction of International Prognostic Index for patients with diffuse large B-cell lymphoma treated with rituximab, cyclophosphamide, doxorubicin, vincristine and prednisone. Leuk Lymphoma 2015;56:2032-8.

17. Li X, Zhang Y, Zhao W, et al. The Glasgow Prognostic Score as a significant predictor of diffuse large $\mathrm{B}$ cell lymphoma treated with R-CHOP in China. Ann Hematol 2015;94:57-63.

18. Porrata LF, Ristow KM, Habermann TM, et al. Peripheral blood absolute lymphocyte/monocyte ratio during rituximab, cyclophosphamide, doxorubicin, vincristine and prednisone treatment cycles predicts clinical outcomes 
in diffuse large B-cell lymphoma. Leuk Lymphoma 2014;55:2728-38.

19. Feng JF, Huang Y, Chen QX. A new inflammation index is useful for patients with esophageal squamous cell carcinoma. Onco Targets Ther 2014;7:1811-5.

20. He X, Zhou T, Yang Y, et al. Advanced Lung Cancer Inflammation Index, a New Prognostic Score, Predicts Outcome in Patients With Small-Cell Lung Cancer. Clin Lung Cancer 2015;16:e165-71.

21. Nozoe T, Ninomiya M, Maeda T, et al. Prognostic nutritional index: a tool to predict the biological aggressiveness of gastric carcinoma. Surg Today 2010;40:440-3.

22. Kanda M, Fujii T, Kodera Y, et al. Nutritional predictors of postoperative outcome in pancreatic cancer. Br J Surg 2011;98:268-74.

23. Go SI, Park S, Kang MH, et al. Clinical impact of prognostic nutritional index in diffuse large $\mathrm{B}$ cell lymphoma. Ann Hematol 2019;98:401-11.

24. Yu W, Guo Q, Wang Z, et al. Clinical Significance of Prognostic Nutritional Index for Patients with Diffuse Large B-cell Lymphoma. Nutr Cancer 2019;71:569-74.

25. Periša V, Zibar L, Knezović A, et al. Prognostic nutritional index as a predictor of prognosis in patients with diffuse large B cell lymphoma. Wien Klin Wochenschr 2017;129:411-9.

26. Yan M, Jurasz $P$. The role of platelets in the tumor microenvironment: From solid tumors to leukemia. Biochim Biophys Acta 2016;1863:392-400.

27. Hu B, Yang XR, Xu Y, et al. Systemic immuneinflammation index predicts prognosis of patients after curative resection for hepatocellular carcinoma. Clin Cancer Res 2014;20:6212-22.

28. Lolli C, Basso U, Derosa L, et al. Systemic immuneinflammation index predicts the clinical outcome in patients with metastatic renal cell cancer treated with sunitinib. Oncotarget 2016;7:54564-71.

29. Passardi A, Scarpi E, Cavanna L, et al. Inflammatory indexes as predictors of prognosis and bevacizumab efficacy in patients with metastatic colorectal cancer. Oncotarget 2016;7:33210-9.

30. Hong X, Cui B, Wang M, et al. Systemic Immuneinflammation Index, Based on Platelet Counts and Neutrophil-Lymphocyte Ratio, Is Useful for Predicting Prognosis in Small Cell Lung Cancer. Tohoku J Exp Med 2015;236:297-304.

31. Mirili C, Paydas S, Kapukaya TK, et al. Systemic immune- inflammation index predicting survival outcome in patients with classical Hodgkin lymphoma. Biomark Med 2019;13:1565-75.

32. Kalweit GA, Feindt P, Micek M, et al. Markers of activated hemostasis and fibrinolysis in patients with pulmonary malignancies: comparison of plasma levels in central venous and pulmonary venous blood. Thromb Res 2000;97:105-11.

33. Pavey SJ, Hawson GA, Marsh NA. Impact of the fibrinolytic enzyme system on prognosis and survival associated with non-small cell lung carcinoma. Blood Coagul Fibrinolysis 2001;12:51-8.

34. Bi XW, Wang L, Zhang WW, et al. High Pretreatment D-Dimer Levels Correlate with Adverse Clinical Features and Predict Poor Survival in Patients with Natural Killer/ T-Cell Lymphoma. PLoS One 2016;11:e0152842.

35. Gao R, Liang JH, Wang L, et al. Low serum cholesterol levels predict inferior prognosis and improve $\mathrm{NCCN}$ IPI scoring in diffuse large B cell lymphoma. Int J Cancer 2018;143:1884-95.

36. Cruz PM, Mo H, McConathy WJ, et al. The role of cholesterol metabolism and cholesterol transport in carcinogenesis: a review of scientific findings, relevant to future cancer therapeutics. Front Pharmacol 2013;4:119.

37. Jensen GL, Cederholm T, Correia MITD, et al. GLIM Criteria for the Diagnosis of Malnutrition: A Consensus Report From the Global Clinical Nutrition Community. JPEN J Parenter Enteral Nutr 2019;43:32-40.

38. Charbonneau B, Maurer MJ, Ansell SM, et al. Pretreatment circulating serum cytokines associated with follicular and diffuse large B-cell lymphoma: a clinic-based case-control study. Cytokine 2012;60:882-9.

39. Hong JY, Ryu KJ, Lee JY, et al. Serum level of CXCL10 is associated with inflammatory prognostic biomarkers in patients with diffuse large B-cell lymphoma. Hematol Oncol 2017;35:480-6.

(English Language Editor: J. Jones)

Cite this article as: Liu T, Ye F, Li Y, Liu A. Comparison and exploration of the prognostic value of the advanced lung cancer inflammation index, prognostic nutritional index, and systemic immune-inflammation index in newly diagnosed diffuse large B-cell lymphoma. Ann Palliat Med 2021;10(9):9650-9659. doi: 10.21037/apm-21-2067 